\title{
Social Media Strategies for Companies: A Comprehensive Framework
}

\author{
Bibian Ogbuji ${ }^{\mathrm{a}}$, Anastasia Papazafeiropoulou ${ }^{\mathrm{b}}$

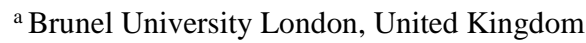 \\ ${ }^{\mathrm{b}}$ Brunel University London, United Kingdom \\ bibian.ogbuji@brunel.ac.uk; \\ anastasia.papazafeiropoulou@brunel.ac.uk
}

\begin{abstract}
Companies when strategizing are looking for innovative ways to have a competitive advantage over their competitors. One way in which they compete is by the adoption of social media. Social media has evolved over the years and as a result, new concepts and applications are developed which promises to provide business value to a company. However, despite the usefulness of social media, many businesses fail to reap its full benefits. The current literature shows evidence of lack of strategically designed process for companies to successfully implement social media. The purpose of this study is to suggest a framework which provides the necessary alignment between social media goals with business objectives. From the literature review, a social media strategy framework was derived to offer an effective step by step approach to the development and implementation of social media goals aligned with a company's business objectives. The contribution to this study is the development of a social media strategy framework that can be used by organisations for business value.
\end{abstract}

Keywords. Social Media, Social Media Strategy, Social Networks, Social Media Platforms

\section{Introduction}

Social media are online platforms that aid global collaboration and sharing amongst users. With the rapid growth and availability of network bandwidth and technology, social media has a significant increase in user count. This has also led to the increase in user-generated content [18]. Hence, it enables users to connect with one another and share information. These platforms are extensively adopted by users of all age group, educational, cultural and economic background [29].

The inarguable growth of social media has allowed profound changes in the environment. Social media is altering the business while introducing development, new learning skills and communication management processes [25]. Social media has changed the traditional marketing methods which is a one-way flow of information (for example, television and radio) while encouraging a two-way communication flow. The use of social media allows easy sharing of information to a wider audience while increasing brand awareness and promoting company's products and services. As a result of this exposure, companies strive to maintain a positive image, have an active online 
presence to communicate with their customers and also satisfy customers' needs. Companies are following the trend of having a presence on social media by creating multiple social media accounts without clear objectives or a strategic approach for its business use. Previous research has shown the implementation of social media is to be done carefully by designing a framework or method to provide the necessary integration between social media and the business goals. Resources such as time, skills, human resource and technology are needed when adopting social media. Therefore, the need to measure return on investment (ROI) arises [25]. Nonetheless, it is a challenge to measure the efficiency and return on investment on a social media approach that has not been strategically designed.

However, so many companies have adopted the use of social media practice in their business. The gap in the literature shows limited availability of social media strategy framework for the implementation of social media for business use. Despite the advantages, some companies are yet to identify clearly what their social media presence entails [18]. Recent research has shown that companies do not have a strategy in the selection of a suitable social media platform for their business [29]. In the absence of a strategic process, a company cannot gain the full benefit that social media provides [15]. Hence, this study aims to address the gap of a lack of a comprehensive social media strategy.

The next section presents the use of social media by companies which leads to the lack of social media strategies in business. Second, we elaborate on existing social media strategies. Third, we discuss the selection process of suitable social media platforms by companies. Fourth, we present a proposed framework. Finally, the conclusion of this study.

\section{Use of social media by Companies}

For many businesses, it is important to engage in social media to benefit from the opportunities it provides. According to Harris and Rae [13], companies have recognised the opportunities that lie in the adoption of social media. For example, it helps build trust and commitment with key stakeholders, employees, and customers.

The different social media platforms such as Facebook, Twitter, LinkedIn, and YouTube are used to enable online interaction share and collect information about products and services. These platforms can serve as a place where different ideas can be collected from a vast range of population concerning the development of a business or its products.

Previous research has shown that social media adoption has created a high positive impact on businesses that incorporates this technology as an innovative process in comparison to firms that are yet to do so [30]. According to $\mathrm{Li} \& \mathrm{Li}$ [24], social media creates a cordial relationship between a company and its customers thereby, influencing various aspects of consumer behaviour which includes purchase behaviour, awareness, information acquisition, post-purchase communication and opinions [28]. According to Jiang et al. [16] from a business perspective, social media can be used in various ways to improve operations and enhance business profile. The process can be done by improved communication, maintaining a good relationship with existing customers and 
attracting new customers, intensify word of mouth effect, advertising company's products and services, improving brand awareness and increasing traffic to websites. Businesses are increasingly attempting to adopt and implement social media as an integral part of work life and to enhance competitive advantage [7].

According to Weisgerber \& Butler [34], social media has enabled organisations to gain exposure to the needs, opinions, and desires of a wider audience and also provide an opportunity for connecting with a highly targeted population that could be potential customers to the organisation. From the organisations' perspective, social media creates a better communication process between customers, stakeholders and the organisation itself which leads to the start of an innovative process in the organisation [23].

\subsection{Lack of Social Media Strategy}

In the early 2000s, during the inception of social media which brought the launch of platforms like Fotolog, Myspace, hi5, Friendster and del.icio.us, companies have established a presence on these platforms to explore the benefits of social media for business. The early adopters were intrigued by the number of users subscribed to these platforms and its increasing usage among its customer base. Previous research has shown that most companies randomly establish a presence on social media while others avoid these platforms altogether because of unknown business risk and lack of understanding of the social media environment [21]. Establishing a presence on several social media platforms without a strategically defined approach does pose some risks to companies. Nevertheless, during the adoption process of social media, companies use up time, human resource, efforts, technology and skills, these parameters need to be put into consideration so the return on investment can be measured accurately. Strategic content planning, resources or undefined job responsibility can lead to the inconstancy of content flow. Therefore, companies will not be able to keep customer's interest for long [26].

In the excitement of creating a presence on social media platform (for example, Facebook page) to enable customers to join (like), or for the display of adverts especially videos which have the tendency to go viral. The video can expose a company in either a positive or negative way; a positive exposure can generate more revenue while a negative exposure can affect the business and the company's reputation [5]. The nature of social media makes it difficult to govern as it is user generated and difficult to control [9]. However, after a careful study of social media mechanisms companies can foresee social media risks and provide quick response to them. Lardi and Fuchs [21] states that the adoption of social media brings an advantage to a company's value when properly incorporated into the company's context. Recent research shows companies are beginning to understand the need to adopt a social media strategy, clearly defining the objectives of the use of having a social media presence for their business, identifying the target audience and the selection of suitable social media platform(s) for specific business needs [21].

A strategic business change impacts all the aspects of the company; people, culture, process, technology and also the implementation of social media strategy. Applying 
social media as a business strategy and not just a platform for marketing allows companies to find an appropriate use for social media with a maximum business value [26].

\section{Existing Social Media Strategy Framework}

Companies are challenged as they face an environment where the adoption rate of social media has massively increased by customers and employees. However, companies face tremendous pressure to create a presence on various platforms as expected by customers [22]. For this reason, they are struggling to implement social media strategically in their business for it is not enough to just create a presence but to use it systematically and enjoy its benefits. However, many companies find implementing a successful social media strategy difficult [4], [18]. There is a few social media strategy that concentrates on different aspects of social media.

Effing and Spil [12] framework provides significant key elements and areas of concern for the development of a social media strategy. The seven elements are as follows; (i) Target audience: companies should define what target group they intend to address using social media. (ii) Channel choice: it is important to select the appropriate choice of channel for different target groups. (iii) Goals: to gain business value, social media should be aligned with business goals. (iv) Resources: adequate resources should be allocated to the success of social media strategy. (v) Policies: should be structured to manage the use of social media in the company. (vi) Monitoring: businesses should listen to what is been said about the companylproducts and services. (vii) Content activities: a clear content post and timeframe are defined to allow regular contribution on the platforms. These elements are derived from the literature review as the components required for a comprehensive social media strategy.

Secondly, Kietzmann et al. [18] framework which is called "The Honeycomb of social media" consist of seven functional building blocks of social media they are: (i) Presence: refers to the availability of users on the platform. (ii) Relationship: is how users relate to one another, (iii) Reputation: is the degree to which users know each other, (iv) Groups: is when users form communities. (v) Identity: this relates to the level of which users reveal themselves, (vi) Conversation: refers to how users communicate with one another, (vii) Sharing: is the exchange of information among users. Hence, these functional building blocks describe the extent in which users engage with social media.

While Kiralova and Pavliceka [19] social media strategy is concerned with using social media as a useful tool for marketing. The paper discussed the competitive advantage gained by the alignment of business strategies with social media in the tourism sector. While Oliveira and Figueira [26] social media strategy concentrated on using social media to develop a functional communication strategy.

The above frameworks do not possess the significant factors needed in a comprehensive social media strategy as they do not contain the necessary requirements for evaluating readiness, development and the implementation of social media in companies. 
The Lardi and Fuchs [21] social media strategy framework forms the core of this study (Fig.1). This framework gives a detailed step by step process on the implementation of social media strategy while considering the goals and objectives of the business.
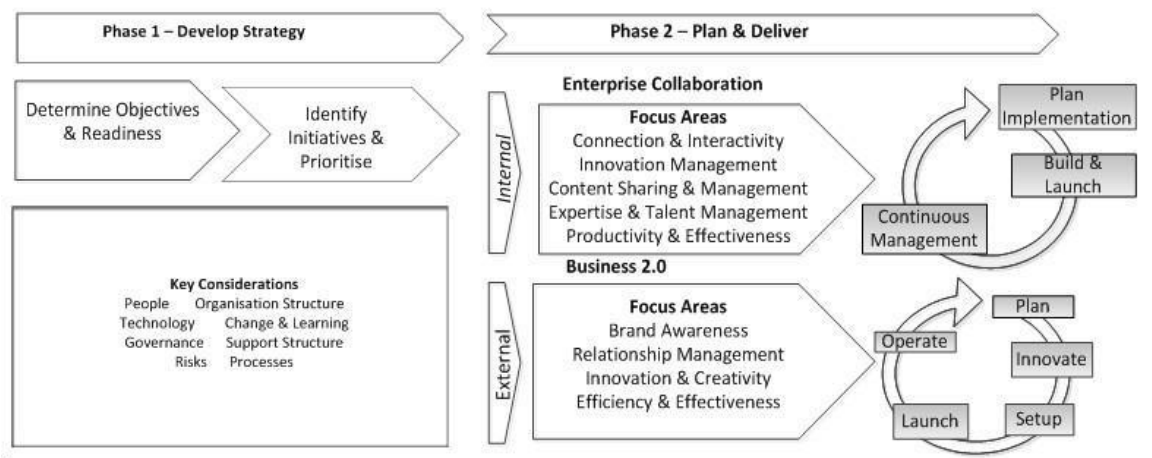

Fig 1. Social Media Strategy Framework [21]

\section{Phase 1 -Development Strategy}

This stage is important as it allows a replete assessment of the company's competence regarding readiness, abilities, objectives and capabilities to adopt social media into their business [16]. This stage also puts into consideration the organisational structure, people, process carried out, governance, changes social media brings to the business process, technology to be adopted and risk involved.

\section{Phase 2 -Plan \& Deliver}

Phase 2 has two parts: Enterprise collaboration (Internal) and Business 2.0 (External).

Enterprise Collaboration: This section focuses on the use of social media within the company. The enterprise collaboration gives power to the employees by slowly getting rigid of the traditional corporate structure in a company. The implementation of enterprise 2.0 creates an opportunity for businesses to tap into the creativity of their employees to enhance productivity and daily work activities [14].

In regards to the focus areas (see Fig. 1) connection and interactivity in the enterprise collaboration is a process of exchange of information. The interactive innovation of social media offers two-way communication, which aids in the easy flow of information between employees to achieve effectiveness and productivity [35]. The continual interaction of employees in enterprise collaboration encourages brainstorming and enhances the chances of the birth of new ideas or innovation.

Enterprise collaboration process is carried out in three stages; (i) Plan implementation: at this stage, companies substantiate the functional requirements, develop a business case that fits the business and employees requirements. Furthermore, they identify a particular business area where social technology can be defined and implemented. Important factors like training for employees, development of unique skills and adoption process are considered critically. (ii) Build and launch: the actual building and 
launching of the social media platform are done at this point. For example, if the company does not want to use existing third-party applications like Facebook etc. they would develop a platform within the corporation to suit their needs (iii) Continuous management: Monitoring and analysing the adopted or developed platform to measure the quality, usefulness. Ongoing analysis of the platform is required for maintenance and future development [21].

Business 2.0: This section handles the external part of the company; customers and stakeholders. This area focuses on using social networking applications in promoting innovative teamwork, interaction with their customers and also collaborating with external stakeholders to produce business innovation [6]. Many companies are at risk because they start the implementation process of Business 2.0 by creating a presence on popularly used social media platforms. It is important to define the purpose of Business 2.0 critically in business by considering the advantage(s) to specific business areas like increasing brand awareness, relationship management, innovation, creativity and also increase efficiency and effectiveness.

There are various social media tools company use for this process. Examples are blogs, microblogging, social networking sites, podcast, etc. [8], [10], [33]. However, the focus area of a company may vary depending on the business area which needs improvement. There are processes outlined for the implementation of Business 2.0 such as; (i) Plan: at the planning stage, a clear identification of the target audience or target group(s) and the social media platforms they use are determined. For example, if a company provides services for different target groups, different messages will be sent to these various groups. Hence, sending a clearly defined message relevant for each target groups is an effective communication strategy to reaching out to customers. Furthermore, the most efficient way to benefit from Business 2.0 initiatives is by the selection of suitable social media platforms [19]. (ii) Innovate: this is where the creative ideas emanate. According to Berman and Hagan [2], innovation is changing the basis of competition, rather than competing heads on with other competitors; companies create new ideas to exploit a growing market need in order have an edge over its competitors and also to gain market advantage. (iii) Setup: before the implementation of Business 2.0 a few adjustments are required to accommodate social media activities in the company. Setup stage is the modification of company's policies and development of social media policies to allow the business handle the necessary change the implementation process brings [12]. Furthermore, social media tools and accounts are setups. The content messages for the target groups and time frame for posting are determined at this point [21]. (iv) Launch: The launch stage is the final stage after the above steps are completed. The launch takes place after the platform(s) are developed and ready for the reveal. The platform(s) can are revealed same day or separate days. The launch approach varies amongst companies. (v) Operate: After the platform(s) have been initiated, the effect of how social media activities are influencing and creating traffic to the corporate websites is monitored [12].

The social media strategy framework is structured in an easy to follow steps. However, it does not include a systemic approach for companies to identify the suitable social media platform to employ for their business. 


\section{Selection of Social Media Platform}

Social media platforms have a vast advantage for social activities hence it is not just a means of sharing information on day-to-day operations; it is a valuable and cost- effective way for businesses to reach their customers. Regardless, it is not appropriate for companies to create an account on all the social media platforms available. Determining the right social media platform(s) for a company is not to be overlooked [11].

Recent studies have shown that companies do not take strategic procedures in selecting suitable social media platform(s) to meet their business values [26]. This has been identified as a gap in the literature. The study for the process of selecting social media platform has little attention in this research area. However, Tavana et al. [31] derived a network structure of factors to consider in the selection process of a suitable social media platform for business. This structure consist of six elements; content, impression score, cost, look \& feel and audience fit. These elements describe the different important factors to consider during the selection process.

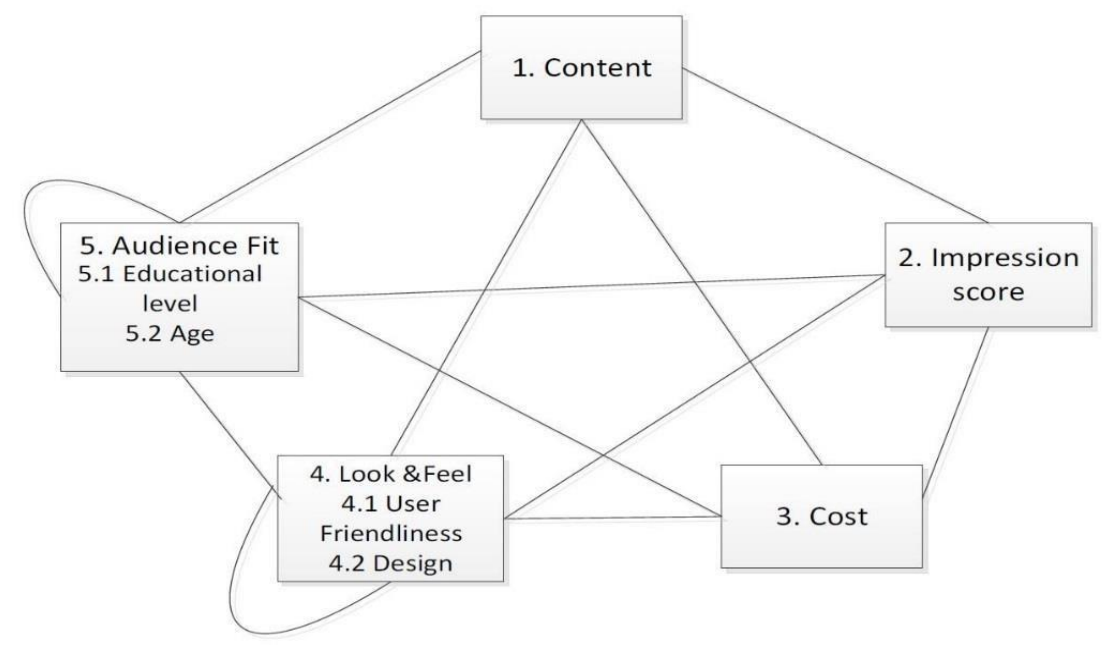

Fig 2. Social Media Platform Evaluation [31]

The social media platform evaluation in figure 2 shows the elements and the interconnection between them. A brief description of the items is discussed below.

Content: With the increased growth of social media in the community, the tools and strategies companies use to communicate with customers have changed appreciably. This significant change allows consumers to generate content online with the intent on sharing and educating one another about products, services, experiences and brands [3]. According to Tavana et al. [31] the content of a social media platform is used to measure the amount of pertinent information on that platform. Hence, content is significant because it can keep customers interested or customers can lose interest then switch to a different platform. Barnes [1] states that it is important to create a schedule for content updates to ensure frequent participation in the platform(s). Kaplan and Haenlein [17] 
indicates that social media is about sharing and interacting with the audience, so it is important to ensure an active presence whereby posting new updates and also engaging in discussions with customers and target audience.

Impression Score: According to Kiralova and Pavliceka [19] research on tourism destination, social media is used as a promotional or marketing tool which has a high number of visitors using social media applications. Therefore in other to monitor these visits impression score is employed. "Impression score is a subjective score used to capture the behaviour of visitors in a site" [31].

Cost: Initially, social media began as a medium for entertainment, then after it became the most common tool for marketing because of the vast advantage it brings to the business area. To create a presence on most social media platforms, this can be done free of charge or at low cost. As a result of it cost- effective nature, it is considered to be the preferred marketing tool in the business environment. Hence, companies have employed social media as an excellent approach and a marketing strategy to reach their customers and potential customers. Companies benefit from cost reduction on marketing by using social media as a marketing strategy [20].

Therefore, multinational companies, small and medium-sized enterprises, non-profitable organisations and even government agencies all implore social media because of it cost effective nature and high level of efficiency [17].

Look and Feel: The look and feel of a site are stated to mean the design and user friendliness of a social media site. Furthermore, it can represent the need for minimal training expense and the simplicity of the tool Tavana et al. [31]. A social media platform with an intuitive and user-friendly interface ensures that customers can engage with the systems without training or difficulty [21].

Audience fit: Companies are advised to define what target groups to address using social media platforms. They have to prioritise their customers to identify the degree of the population who use and access social media [12]. According to Edwards [11], the most effectual way for a company to reach its target audience is by identifying its business goals and objectives and the audience suitable for the business. For example, age group, educational level, gender, etc. all of these determines an audience fit. Therefore, if a company is interested in the young audience, a good example of social media platform to concentrate on would be snap chat and Instagram.

Having seen Fig. 1 and Fig. 2, this study has merged both structures to form a comprehensive social media strategy framework. 


\section{$5 \quad$ Proposed Framework}

A gap from the literature suggests that there is a lack of focus on the capability and readiness for companies to adopt social media. The literature states a limitation in research on social media strategy frameworks for business use and the evaluation of social media platform. The merging of social media strategy frameworks in Fig. 1 and the social media platform evaluation in Fig. 2 address their independent limitations. As discussed in the social media strategy framework in Fig. 1, the framework considered the readiness of an organisation to adopt social media and how it is used in specific business context. However, it lacks a systemic approach for selection of social media platform(s) that is suitable for its business which Fig. 2 addresses by merging both frameworks. While studying Fig 1 and Fig. 2 independently, it was considered that the frameworks were incomplete because they do not contain the necessary factors needed for the implementing of social media strategy in an organisation. The existing frameworks as discussed in section 3 have some limitations which the comprehensive social media strategy in Fig 3 addresses. For example "The Honeycomb Framework" [18] is designed to help understand social media and its functionalities, so this does not necessarily focus on social media readiness or how it can be implemented successfully in a company [27].

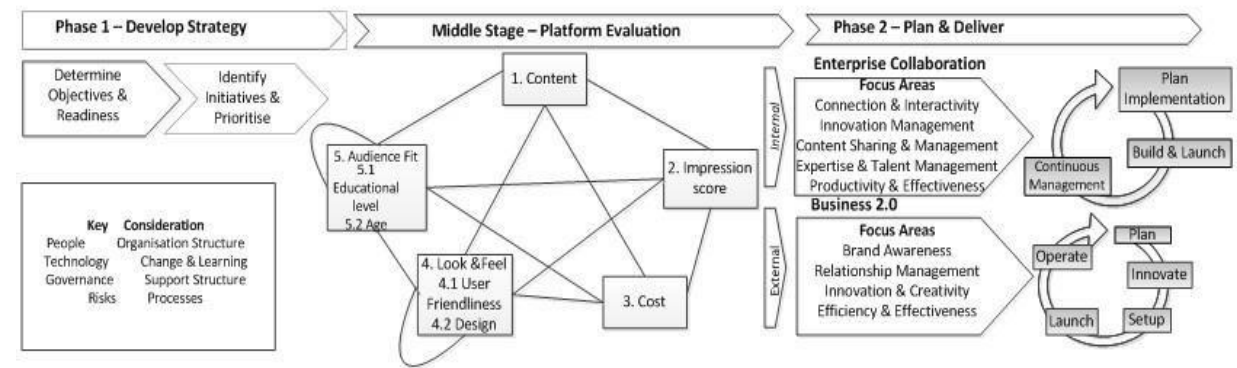

Fig 3. A Comprehensive Social Media Strategy Framework

The social media strategy framework (see Fig.1) gives a detailed illustration on how social media can be implemented by a company effectively. While considering the company's readiness, transparency to employees and customers, the merging between social media and existing business activities and the importance of defining the target audience for social media use [32]. Nonetheless, the social media strategy framework (Fig.1) lacks a profound process to select a suitable platform for both the Enterprise Collaboration (internal) and Business 2.0 (external) which the framework in Fig. 2 addresses.

The Platform evaluation process (see Fig. 2) shows the key factors necessary for consideration in the evaluation process for selecting a suitable social media platform. The literature shows there is a lack of a framework to determine the right social media 
platform for businesses. Although Edwards [11] suggested ways in selecting the right social media platforms by having a good knowledge of user statistics such as daily active users, monthly active users and overall users of each platform. More so, gave an explanation of how these platforms can be used, however, this is not an efficient way to determine the precise social media platform for it is based on number counts. Different platforms are developed for a different purpose with distinct functionality and separate target audience [32]. For example, LinkedIn is known to be a platform for work professionals.

From Fig.1 and Fig.2 forms the comprehensive framework for social media strategy implementation and platform evaluation process. This framework provides the necessary need for adoption and improvement of an existing social media practice and the useful method for the selection of social media platform. The framework was merged and is presented in Fig. 3. We suggest this framework as a comprehensive way for the adoption and implementation of social media in companies.

\section{Conclusion}

The continual development of information technology brings both opportunities and challenges in the world. Managers are faced with the technologically progressive environment. Hence, social media networks have become a necessary tool for business. Social media creates a platform for companies through which they can improve their business and customer experience. Social media provide several opportunities but also come with some risks. Companies need to evaluate which social media platform is appropriate for its business. It is important for businesses to select the best few platforms that suit its company's need. Companies need a social media strategy to help adopt and implement social media appropriately to their business. The suggested framework aids in this regard as it presents a mechanism, which various companies such as, the service companies can use to identify what areas of the business need improvement and how best to exploit social media for the business purpose. Thus, intensifying the likelihood of gaining a competitive advantage. The framework is also contributing to the advancing of knowledge in the domain of social media adoption from the company's perspective. As the current literature is not providing any theoretical frameworks in the application of social media strategies by companies our work is offering a practical well as a theoretical tool in this domain.

\section{References}

1. Barnes, I.: Twitter in special libraries: a distributed social media strategy. Public Services Quarterly 10(1), 62-65 (mar 2014), http://www.tandfonline.com/doi/full/10.1080/15228959.2014.875789http://www.tand fonline.com/doi/abs/10.1080/15228959.2014.875789

2. Berman, S.J., Hagan, J.: How technology-driven business strategy can spur innovation and growth. Strategy \& Leadership 34(2), 28-34 (2006) 
3. Blackshaw, P., Nazzaro, M.: Consumer-generated media (cgm) 101: Word-of-mouth in the age of the web-fortified consumer. New York: Nielsen BuzzMetrics (2006)

4. Bottles, K., Sherlock, T.: Who should manage your social media strategy. Physician Executive 37(2), 68-72 (2011)

5. Brodie, R.J., Ilic, A., Juric, B., Hollebeek, L.: Consumer engagement in a virtual brand community: An exploratory analysis. Journal of Business Research 66(1), 105-114 (2013)

6. Burrus, D.: Social networks in the workplace: the risk and opportunity of business 2.0. Strategy \& Leadership 38(4), 50-53 (2010)

7. Carmichael, D., Cleave, D.: How effective is social media advertising? A study of Facebook Social Advertisements. In: Internet Technology and Secured Transactions, 2012 International Conference for. pp. 226-229 (2012)

8. Chiang, H.S.: Continuous usage of social networking sites: The effect of innovation and gratification attributes. Online Information Review 37(6), 851-871 (2013)

9. Dijkmans, C., Kerkhof, P., Beukeboom, C.J.: A stage to engage: Social media use and corporate reputation. Tourism Management 47, 58-67 (2015)

10. Dioko, L., Harrill, R., María Munar, A.: Tourist-created content: rethinking destination branding. International Journal of Culture, Tourism and Hospitality Research 5(3), 291-305 (2011)

11. Edwards, V.: Determining the right social media networks for your business. Journal of Digital \& Social Media Marketing 3(3), 217-228 (2015)

12. Effing, R., Spil, T.A.M.: The social strategy cone: Towards a framework for evaluating social media strategies. International Journal of Information Management 36(1), 1-8 (feb 2016), http://www.sciencedirect.com/science/article/pii/S0268401215000778

13. Harris, L., Rae, A.: Social networks: the future of marketing for small business. Journal of business strategy (2009), http://www.emealdinsight.com/doi/pdf/10.1108/02756660910987581

14. Husin, B., Heikal, M., Swatman, P.M.C., bin Husin, M.H., Swatman, P.M.C.: Removing the barriers to Enterprise 2.0. In: Technology and Society (ISTAS), 2010 IEEE International Symposium on. pp. 275-283 (2010), http://ieeeplore.ieee.org/xpls/abs $\left\{\begin{array}{l}\text { \} } \\ \text { all.jsp?arnumber }=5514627\end{array}\right.$

15. Jansen, B.J., Zhang, M., Sobel, K., Chowdury, A.: Twitter power: Tweets as electronic word of mouth. Journal of the American society for information science and technology 60(11), 2169-2188 (2009)

16. Jiang, H., Luo, Y., Kulemeka, O.: Leading in the digital age: A study of how social media are transforming the work of communication professionals. Telematics and Informatics 33(2), 493-499 (oct 2016), http://www.sciencedirect.com/science/article/pii/S0736585315001264

17. Kaplan, A.A.M., Haenlein, M.: Users of the world, unite! The challenges and opportunities of Social Media. Business horizons 53(1), 59-68 (jan 2010), http://www.sciencedirect.com/science/article/pii/S0007681309001232http://www.sciencedirect.com/science/article/pii/S0007681309001232,

18. Kietzmann, J.J.H., Hermkens, K., McCarthy, I.P.I., Silvestre, B.S.B.: Social media? Get serious! Understanding the functional building blocks of social media. Business horizons 54(3), 241-251 (2011), http://www.sciencedirect.com/science/article/pii/ S0007681311000061 
19. Kiralova, A., Pavliceka, A.: Development of Social Media Strategies in Tourism Destination. Procedia - Social and Behavioral Sciences 175, 358-366 (feb 2015), http://www.sciencedirect.com/science/article/pii/S1877042815012719

20. KirtiÅ§, A.K., Karahan, F.: To Be or Not to Be in Social Media Arena as the Most Cost-Efficient Marketing Strategy after the Global Recession. Procedia - Social and Behavioral Sciences 24, 260-268 (2011), http://www.sciencedirect.com/science/ article/pii/S1877042811016119

21. Lardi, K., Fuchs, R.: Social media strategy. vdf Hochschulverlag AG (2013)

22. Larson, K., Watson, R.: The value of social media: toward measuring social media strategies (2011)

23. Lehmkuhl, T., Baumol, U., Jung, R.: Towards a maturity model for the adoption of social media as a means of organizational innovation. In: System Sciences (HICSS), 2013 46th Hawaii International Conference on. pp. 3067-3076. IEEE (2013)

24. Li, S., Li, J.: Linking social media with open innovation: an intelligent model. . . . Technology and Automation (ICICTA), 2014 7th ... (2014),

http://ieeexplore.ieee.org/xpls/abs\{_\}all.jsp?arnumber=7003550

25. M. Schroeder, H.: Social media in business strategy: the learning and development implications. Development and Learning in Organizations: An International Journal 28(6), 12-15 (2014)

26. Oliveira, L., Figueira, Á.: Benchmarking analysis of social media strategies in the higher education sector. Procedia Computer Science 64, 779-786 (2015)

27. Omosigho, O.E., Abeysinghe, G.: Evaluating readiness of organizations to adopt social media for competitive advantage. In: Information Society (i-Society), 2012 International Conference on. pp. 16-21. IEEE (2012)

28. Paniagua, J., Sapena, J.: Business performance and social media: Love or hate? Business Horizons 57(6), 719-728 (nov 2014), http://www.sciencedirect.com/science/ article/pii/S0007681314000974

29. Persaud, A., Spence, M., Rahman, M.: Social media implementation in small service firms. International Journal of E-Business Development (2012)

30. Smith, F., Taglino, F.: Semantics-based social media for collaborative open innovation. In: Collaboration Technologies and Systems (CTS), 2014 International Conference on. pp. 345-352 (2014),

http://ieeexplore.ieee.org/xpls/abs\{_\}all.jsp?arnumber $=6867587$

31. Tavana, M., Momeni, E., Rezaeiniya, N., Mirhedayatian, S.M., Rezaeiniya, H.: A mnovel hybrid social media platform selection model using fuzzy ANP and COPRASG. Expert Systems with Applications 40(14), 5694-5702 (2013)

32. Value, B.: Quarterly executive (2010)

33. Viégas, F.B.: Bloggers' expectations of privacy and accountability: An initial survey. Journal of Computer-Mediated Communication 10(3), 00-00 (2005)

34. Weisgerber, C., Butler, and S.H.: Social media as a professional development tool: using blogs, microblogs, and social bookmarks to create personal learning networks. Cutting-edge Technologies in Higher Education 3, 339-363 (2011)

35. Zolkepli, I.A., Kamarulzaman, Y.: Social media adoption: The role of media needs and innovation characteristics. Computers in Human Behavior 43, 189-209 (2015) 